\title{
Evaluation of recombinant multi-epitope proteins for diagnosis of goat schistosomiasis by enzyme-linked immunosorbent assay
}

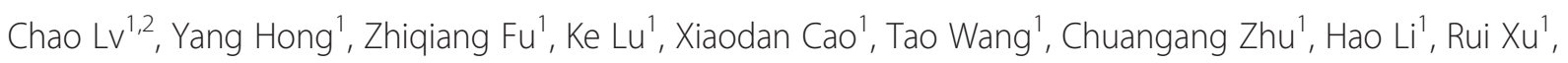
Bingguang Jia', Qian Han ${ }^{1}$, Xuefeng Dou ${ }^{1,2}$, Yuanxi Shen ${ }^{1,2}$, Zuhang Zhang ${ }^{1}$, Jinli Zai ${ }^{1}$, Jintao Feng ${ }^{1}$ and Jiaojiao $\operatorname{Lin}^{1,3^{*}}$

\begin{abstract}
Background: Schistosomiasis is a huge threat to human and animal health. Apart from bovines, goats play an important role in the transmission of schistosomiasis in some endemic areas of China. An accessible, quality-assured goat schistosomiasis diagnostic technique is needed. Recently, our laboratory identified two recombinant diagnostic antigens, SjPGM and SjRAD23 via an immuno-proteomic method. The application of these two recombinant antigens to develop a higher sensitivity and specificity technique for the sheep schistosomiasis diagnosis is urgently needed.

Methods: Epitopes of SjPGM and SjRAD23 were predicted and three polypeptides, two from SjRAD23 and one from SjPGM, were selected. Recombinant plasmids containing two to three DNA sequences encoding predicted polypeptides or large hydrophilic region of Sj23 (LHD-Sj23) were constructed and expressed. Eight recombinant schistosome antigens including four multi-epitope proteins and four recombinant single-molecule antigens as well as SEA, were assessed by ELISA in 91 sera from schistosome-infected goats, 44 sera from non-infected goats, 37 sera from Orientobilharzia-infected goats, and 12 from Haemonchus contortus-infected goats.

Results: ELISA tests showed that three multi-epitope proteins had higher sensitivity than the four single-molecule antigens (rSjRAD23, rSjPGM, rBSjRAD23-1, rBSj23) and the multi-epitope protein rBSjPGM-BSjRAD23-1-BSj23 had the highest sensitivity $(97.8 \%, 89 / 91)$ and maintained good specificity $(100 \%, 44 / 44)$ as well as low cross-reactivity with haemonchosis $(8.33 \%, 3 / 12)$ and orientobilharziasis $(13.51 \%, 5 / 37)$ in the diagnosis of goat schistosomiasis. In contrast, when SEA was applied as a diagnosis antigen, it had $100 \%$ (91/91) sensitivity, 75 \% (33/44) specificity, 25 and $83.78 \%$ cross-reactivity with haemonchosis (3/12) and orientobilharziasis (31/37), respectively.

Conclusions: The application of recombinant multi-epitope proteins may increase the sensitivity of diagnosis technique and retain high specificity of single-molecule antigens for schistosomiasis, and the recombinant antigen rBSjPGM-BSjRAD23-1-BSj23 has the potential to be used as a diagnosis antigen for goat schistosomiasis.
\end{abstract}

Keywords: Recombinant multi-epitope proteins, Diagnosis, Goat schistosomiasis, ELISA

\footnotetext{
* Correspondence: jjlin@shvri.ac.cn

${ }^{1}$ Key Laboratory of Animal Parasitology, Ministry of Agriculture of China,

Shanghai Veterinary Research Institute, Chinese Academy of Agricultural Sciences, Shanghai, China

${ }^{3}$ Jiangsu Co-innovation Center for Prevention and Control of Important

Animal Infectious Diseases and Zoonoses, Yangzhou, China

Full list of author information is available at the end of the article
} 


\section{Background}

Schistosomiasis japonica is a major parasitic zoonosis in China and Southeast Asia, which seriously threatens human and animal health in endemic areas. At the end of 2013, there were still 184,943 cases of schistosomiasis, including 29,796 cases of advanced schistosomiasis and 1700 fatal cases in China [1]. Although Schistosoma japonicum is known to infect more than 40 species of mammals in addition to humans, schistosome-infected bovines are considered to be the main host reservoir in China; however, in some marshland endemic areas goats are also reported to be a major infection source $[2,3]$. There are 1,033,056 cattle and buffaloes, 2,024,512 goats and sheep and 891,301 other domestic animals, estimated to breed in schistosomiasis endemic regions in China in 2012 [4]. A survey from 2005 to 2010 in the Dongting lake region indicated that the proportion of the schistosome-infected bovines was 23.5-58.2 \% of total infected ruminants, while there were 41.8-76.5\% infected goats and infection rates in cattle and goats were significantly higher than those found in water buffaloes in the survey years [5]. The studies mentioned above showed both bovines and goats play significant roles in disease transmission in some endemic areas of China. Great achievements in domestic animal schistosomiasis control have been attained over the past six decades in China, the prevalence and intensity of schistosome infection have significantly decreased both in humans and domestic animals [6]. The development of a more sensitive and specific diagnostic technique is urgently required in the low endemic areas for case detection, surveillance and screening of target individuals for treatment [7].

Although the pathogen detection techniques that include stool egg examination and miracidium hatching are the gold standard methods to diagnose domestic animal schistosomiasis, serological diagnosis has its advantage with high sensitivity and easy operation, and has been widely applied in disease diagnosis.

Nowadays, schistosome soluble egg antigen (SEA) has been employed as the diagnosis antigen in most developed serological detecting methods. When applied in detection, SEA usually possesses higher sensitivity than other native parasite antigens, such as adult worm antigen or cercariae antigen, and at the same time has lower specificity, higher cross-reactivity with other parasite infections, and specific antibodies drop slowly post chemotherapy. For increasing the specificity of the diagnosis method, screening of single molecules with potential as diagnosis antigen has been carried out via enzymelinked immunosorbent assay (ELISA), chip technology, pull-down, immune-proteomics and other techniques. Several defined antigens have been identified, their recombinant proteins have been prepared and the effects of these proteins as diagnostic molecules have been evaluated. Generally speaking, most of the purified recombinant single-molecule antigens have shown higher specificity, lower cross-reactivity, but poor sensitivity when compared with those of SEA. Recently, our laboratory has identified two recombinant proteins, rSjPGM (phosphoglycerate mutase) and rSjRAD23 (radiationsensitive protein) which possess value for schistosomiasis diagnosis by immunoproteomics technology. Our previous studies also showed that rSjPGM and rSjRAD23 were promising diagnostic antigens for water buffalo schistosomiasis [8].

It is speculated that a good way to improve the sensitivity of the diagnostic method may be by constructing and applying multi-epitope recombinant antigens as a diagnosis antigen in place of a single-molecule antigen [9-11]. In our previous study we constructed an epitope recombinant protein pGEX-Sj23-SjGCP and ELISA tests showed that the recombinant antigen was a promising diagnostic antigen for detecting $S$. japonica in water buffalo better than signal recombinant protein $\mathrm{Sj} 23$ and SjGCP [9]. In this study, we constructed two bivalent epitope recombinant antigens and two trivalent epitope recombinant antigens based on the epitope analysis of three schistosomiasis diagnosis antigens Sj23, SjPGM and SjRAD23, and parallelly compared the sensitivity and specificity of these newly constructed multi-epitope recombinant antigens with the relative single-molecule recombinant antigens, rSjPGM, rSjRAD23, rLHD-Sj23 and SEA using them as diagnostic antigens for detecting goat schistosomiasis by ELISA. The purpose of this study was to screen a promising diagnosis antigen and establish a more sensitive technique for the diagnosis of goat schistosomiasis.

\section{Methods \\ Ethical approval}

All animal care procedures were conducted in strict accordance with the Regulations for the Administration of Affairs Concerning Experimental Animals (Date issued: 1988.11.1), and all efforts were made to minimise suffering. The infection of goats and rabbits with S. japonicum and the sera or livers collection as well as others animal procedures were approved by the Animal Care and Use Committee of Shanghai Veterinary Research Institute, Chinese Academy of Agricultural Sciences for the use of laboratory animals (Permit ID Number: SHVRI 2013-0309).

\section{Animals}

Male New Zealand rabbits $(2.0-2.5 \mathrm{~kg})$ were bought from the Shanghai Experimental Animal Center, Chinese Academy of Sciences. 


\section{Goats sera}

Ninety-one schistosome positive sera were obtained from goats artificially infected with $S$. japonicum and 44 sera from uninfected animals were collected from goats from schistosomiasis non-endemic areas. Totals of 37 Orientobilharzia-positive sera were collected from goats in which parasites were found in their portal venous system in Nyêmo County, XiZang and 12 Haemonchus contortus-positive sera were collected from goats in which parasites were found in their abomasa. Serum $(10 \mu \mathrm{l})$ was taken from each of 20 schistosome-positive or negative sera and mixed as reference positive or negative sera, respectively. All sera were stored at $-80{ }^{\circ} \mathrm{C}$ in the laboratory until use.

\section{Preparation of SEA}

The SEA were prepared as previously described $[12,13]$. Briefly, after the rabbits were infected artificially with $S$. japonicum for 42 days, the livers were collected and stored at $4{ }^{\circ} \mathrm{C}$ for one day. Then, the livers were homogenised in phosphate buffered saline (PBS). The mixture was sequentially passed through $60,80,120,160$, and 200 mesh screens to separate the eggs from liver tissues. The collected solution was centrifuged at $5000 \times g$ for $10 \mathrm{~min}$. The precipitation was washed three times with PBS. The pellet was resuspended with $100 \mathrm{ml}$ PBS containing 7.5 $\mathrm{U}$ trypsin and was incubated at $37{ }^{\circ} \mathrm{C}$ for $2 \mathrm{~h}$ in constant temperature shaker to remove the remaining host proteins. Then, the solution was centrifuged at $5000 \times g$ for $2 \mathrm{~min}$ and the upper liver paste was removed. The pellet was resuspended with PBS, centrifuged as previously and the upper liver paste was removed; this procedure was repeated several times until a uniform golden yellow precipitation was obtained. The qualities of the eggs were examined under the microscope. The purified eggs were diluted with suitable amounts of PBS. Freezing and thawing was repeated for three times and then sonicated using an ultrasonic cell crusher on ice. The sample was centrifuged at $12,000 \times g$ for $20 \mathrm{~min}$. The supernatant was collected as SEA.

\section{Epitope analysis of SjPGM, SjRAD23 and LHD-Sj23}

A series of prediction tools were used to select the epitopes of SjPGM (GenBank accession no. FN315287) and SjRAD23 (GenBank accession no. FN314619). The online software, BepiPred 1.0 was chosen to predict linear B-cell epitopes [14]. The antigenicity, surface accessibility prediction and long loops prediction were analysed by using PredictProtein and IEDB Analysis Resource [15, 16]. The T-epitope Designer and IEDB Analysis Resource were used to predict T-cell epitopes [17]. The peptides with the highest score in each software were selected. The B-cell epitopes and antigenicity of the predicted proteins were considered first. The peptides which included most high score B-cell epitopes, possessing higher antigenicity and corresponding to most or part of other prediction results were chosen as the SjPGM or SjRAD23 epitopes or epitope-rich regions.

Two epitopes were selected from SjRAD23, named as BSjRAD23-1 and BSjRAD23-2, respectively. One epitope was selected from SjPGM and named BSjPGM.

The large hydrophilic region of Sj23 (LHD-Sj23) (GenBank accession no. FN323147) was selected as a candidate fragment. Previous studies have shown that the LHD-Sj23 had a high immunogenicity and was rich region of B-cell and T-cell epitopes $[18,19]$. It was also shown that the LHD-Sj23 is a good candidate antigen for detecting cattle and goat schistosomiasis [20]. The LHD-Sj23 was named BSj23.

\section{Construction of recombinant multi-epitope expression plasmids}

The primer sets containing specific restriction enzyme sites (Table 1) were designed to amplify and fuse a desired segment against the cDNA sequence of each peptide. PCRs were performed using the cDNA of 42-day S. japonicum as a template. Fifty $\mu \mathrm{l}$ of reaction mixture contained $5 \mu \mathrm{l}$ of buffer, $4 \mu \mathrm{l}$ of $2.5 \mathrm{mM}$ dNTP, $4 \mu \mathrm{l}$ of each $10 \mathrm{nmol} /$ $\mu \mathrm{l}$ primer, $0.5 \mu \mathrm{l}$ of $5 \mathrm{u} / \mu \mathrm{l}$ Ex Taq DNA polymerase (Takara,

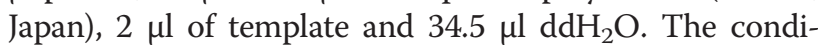
tions for PCR were as follows: $95{ }^{\circ} \mathrm{C}$ for $5 \mathrm{~min}$, followed by 34 cycles of $94{ }^{\circ} \mathrm{C}$ for $1 \mathrm{~min}, 55^{\circ} \mathrm{C}$ for $30 \mathrm{~s}, 72{ }^{\circ} \mathrm{C}$ for $1 \mathrm{~min}$, and a final extension of $72{ }^{\circ} \mathrm{C}$ for $10 \mathrm{~min}$. The PCR was performed using $\mathrm{T} 100^{\mathrm{rm}}$ Thermal Cycler (BIO-RAD, USA). The PCR products were purified using Mini-DNA fragment rapid purification kit (BioDev, China).

The purified PCR products were digested with their respective restriction enzymes and inserted into the pET28a $(+)$ or pET32a $(+)$ vector (Novagen, USA). For example, to construct BSjPGM-BSj23/ pET28a (+), the nucleotide sequence of BSjPGM was digested with BamHI and EcoRI, and inserted into pET28a (+). Then $\mathrm{BSjPGM} / \mathrm{pET} 28 \mathrm{a}(+)$ and the nucleotide sequence of BSj23 were digested with EcoRI and HindIII, then linked using T4 DNA ligase (Takara, Japan) to construct the BSjPGM-BSj23/pET28a (+). The other multi-epitope expression plasmids were constructed with the same method. All recombinant fragments were inserted into pET-28a (+) except BSj23 which was inserted into pET$32 \mathrm{a}(+)$. The constructed multi-epitope plasmids were transformed into Escherichia coli BL21 (DE3) (Beijing TransGen Biotech, China). All the multi-epitope plasmids were verified by PCR and restriction enzyme digestion as well as sequencing.

Expression and purification of the recombinant antigens Escherichia coli cells containing constructed plasmids were cultured in Luria-Bertani broth containing kanamycin 
Table 1 Primer information for cDNA encoding multi- epitope antigens

\begin{tabular}{|c|c|c|c|c|}
\hline CDNA & Primer & & Sequence $^{a}$ & Enzyme \\
\hline \multirow[t]{2}{*}{ BSjRAD23-1 } & forward & & 5'-CGCGAATTCATACATTCAGGCAAGG-3' & EcoRl \\
\hline & reverse & & 5'-CGCAAGCTTGGGTAGGCTAGGCT-3' & Hindlll \\
\hline \multirow[t]{2}{*}{ BSj23 } & forward & & 5'-CGCGAATTCATGACTGGTGCTCTGGA-3' & EcoRl \\
\hline & reverse & & 5'-CGCAAGCTTCTAGTTGCGTTTTAAG-3' & Hindlll \\
\hline \multirow[t]{4}{*}{ BSjPGM-BSj23 } & BSjPGM & forward & 5'-CGCGGATCCTGGCGTCTAAATGAAAGA-3' & $\mathrm{BamHI}$ \\
\hline & & reverse & 5'-CGCGAATTCAAACCAGAATGGTAGT-3' & ECORI \\
\hline & BSj23 & forward & 5'-CGCGAATTCATGACTGGTGCTCTGGA-3' & EcoRI \\
\hline & & reverse & 5'-CGCAAGCTTCTAGTTGCGTTITAAG-3' & Hindlll \\
\hline \multirow[t]{4}{*}{ BSjPGM-BSjRAD23-1 } & BSjPGM & forward & 5'-CGCGGATCCTGGCGTCTAAATGAAAGA-3' & $\mathrm{BamHI}$ \\
\hline & & reverse & 5'-CGCGAATTCAAACCAGAATGGTAGT-3' & EcoRl \\
\hline & BSjRAD23-1 & forward & 5'-CGCGAATTCATACATTCAGGCAAGG-3' & EcoRl \\
\hline & & reverse & 5'-CGCAAGCTTGGGTAGGCTAGGCT-3' & HindllI \\
\hline \multirow[t]{2}{*}{ BSjPGM-BSjRAD23-1-BSj23 } & BSj23 & forward & 5'-CGCAAGCTTATGACTGGTGCTCTGGA-3' & Hindlll \\
\hline & & reverse & 5'-CGCCTCGAGCTAGTTGCGTITAAG-3' & Xhol \\
\hline \multirow[t]{6}{*}{ BSjRAD23-2-BSjPGM-BSj23 } & BSjRAD23-2 & forward & 5'-CGCGGATCCATGGTCATACGAGCAATG-3' & BamHI \\
\hline & & reverse & 5'-CGCGAGCTCTGCGATTGGGTCTTCTGA-3' & Sacl \\
\hline & BSjPGM & forward & 5'-CGGGAGCTCTGGCGTCTAAATGAAAG-3' & Sacl \\
\hline & & reverse & 5'-CGCAAGCTTAAACCAGAATGGTAG-3' & Hindlll \\
\hline & BSj23 & forward & 5'-CGCAAGCTTATGACTGGTGCTCTGGA-3' & Hindlll \\
\hline & & reverse & 5'-CGCCTCGAGCTAGTTGCGTITTAAG-3' & Xhol \\
\hline
\end{tabular}

${ }^{a}$ The sequences of the restriction enzymes are underlined

$(1 \mathrm{mM})$, in a shaker at $37^{\circ} \mathrm{C}$. Isopropyl- $\beta$-D-thiogalactopyranoside $(1 \mathrm{Mm})$ was added when the $\mathrm{OD}_{600}$ was around 0.6 and cultures were grown for a further $6 \mathrm{~h}$ at $37^{\circ} \mathrm{C}$ with agitation $(250 \mathrm{rpm})$. Bacterial cells were then centrifuged at $12,000 \times g$ for $10 \mathrm{~min}$ and then resuspended in $10 \mathrm{ml}$ PBS. Purification of recombinant proteins was applied by His •Bind Resin chromatography (Novagen, USA) following the manufacturer's instructions. These his-tagged fusion proteins purified by affinity chromatography were analysed by sodium dodecyl sulfate-polyacrylamide gel electrophoresis (SDS-PAGE). The purified inclusion body proteins were dialyzed against PBS solution. The fusion proteins of SjPGM and SjRAD23 were obtained and purified as described above [8]. The concentration of purified recombinant proteins was measured using the BCA Protein Assay (Sangon Biotech, China).

\section{Comparison of the sensitivity and specificity of different recombinant antigens in goat schistosomiasis diagnosis by ELISA}

Based on checkerboard titration analysis, microtiter plates (Costar, USA) were coated overnight at $4{ }^{\circ} \mathrm{C}$ with $100 \mu \mathrm{l}$ per well containing $15 \mu \mathrm{g} / \mathrm{ml}$ of each tested recombinant antigen except $5 \mu \mathrm{g} / \mathrm{ml}$ for rBSjPGM-BSj23, or $15 \mu \mathrm{g} / \mathrm{ml} \mathrm{SEA}$ diluted in carbonate bicarbonate buffer ( $\mathrm{pH}$ 9.6). Plates were blocked with $1 \%$ gelatin for $1 \mathrm{~h}$ at $37^{\circ} \mathrm{C}$. Sera at a dilution of 1:100 with PBST (PBS with
$0.05 \%$ Tween 20$)$ was added to the wells $(100 \mu \mathrm{l} /$ well $)$ and incubated for $1 \mathrm{~h}$ at $37^{\circ} \mathrm{C}$. Each serum was repeated for three wells in one test. Horseradish peroxidase-conjugated rabbit anti-goat immunoglobulin G (Santacruz, USA) diluted in 1:6000 (1:4000 for rBSjRAD23-1 and rBSjPGM-BSjRAD23-1) with PBST was added (100 $\mu \mathrm{l} /$ well) and incubated at $37{ }^{\circ} \mathrm{C}$ for $45 \mathrm{~min}$. The plates were washed three times with PBST. 3,3',5,5' - Tetramethyl benzidine dihydrochloride was added to each plates $(100 \mu \mathrm{l} /$ well $)$ and reactions were stopped $10 \mathrm{~min}$ later using $2 \mathrm{M}$ sulfuric acid $(50 \mu \mathrm{l} /$ well). Optical density (OD) at $450 \mathrm{~nm}$ was determined using a microplate reader (BioTek, USA). Each ELISA reaction was performed with a reference negative serum control and a reference positive serum control.

\section{Statistics}

The 2.1 times of the mean absorbance value of the reference negative sera was set as the cutoff value. A sample was considered positive when its mean absorbance value was higher than the cutoff value. Statistical analysis was performed using Stata software (version 13/SE) and $95 \%$ confidence intervals $(\mathrm{CI})$ were determined for the sensitivity, specificity and cross-reactivity of each test. Sensitivity and specificity were calculated as follows: sensitivity $=$ no. of true positives/(no. of true positives + no. of false negatives); specificity $=$ no. of true negatives $/$ (no. 
of true negatives + no. of false positives). Sensitivity, specificity and cross-reactivity for recombinant proteins were compared with that of SEA.

\section{Results}

Selection and cloning of CDNA encoding epitope-rich peptides from SjPGM and SjRAD23

Taking all of the analysis results from each predictive tool into consideration (Table 2), one polypeptide was selected from SjPGM and named BSjPGM, and two polypeptides were selected from SjRAD23 and named BSjRAD23-1 and BSjRAD23-2. BSjPGM contained 82 amino acids encoding the 85 to 166 amino acid sequence of SjPGM with two B-cell epitopes and two Tcell epitopes. BSjRAD23-1 and BSjRAD23-2 contained 78 amino acids and 65 amino acids, encoding amino acids 46-123 and 166-230 of the sequence of SjRAD23, with one and two B-cell epitopes as well as one and two T-cell epitopes respectively. The nucleotide sequence encoding BSjPGM, BSjRAD23-1 and BSjRAD23-2 were amplified (Fig. 1), sequenced and sub-cloned into pET-28a $(+)$.

\section{Construction of recombinant multi-epitope expression plasmids}

A total of nine expression plasmids were successfully constructed: BSjPGM/pET-28a (+); BSjRAD23-1/pET-28a (+): BSjRAD23-2/pET-28a (+); BSj23/pET-32a (+); BSjPGMBSj23/pET-28a(+); BSjPGM-BSjRAD23-1/pET-28a (+); BSjPGM-BSjRAD23-1-BSj23/pET-28a (+); BSjRAD23-2BSjPGM-BSj23/pET-28a(+); and BSjRAD23-2-BSjPGM/ pET-28a $(+)$. The SjPGM/pET-28a (+) and SjRAD23/pET28a $(+)$ plasmids were constructed according to a previous study [6]. The successful construction of recombinant multi-epitope plasmids were verified by PCR and sequenced (Fig. 2).

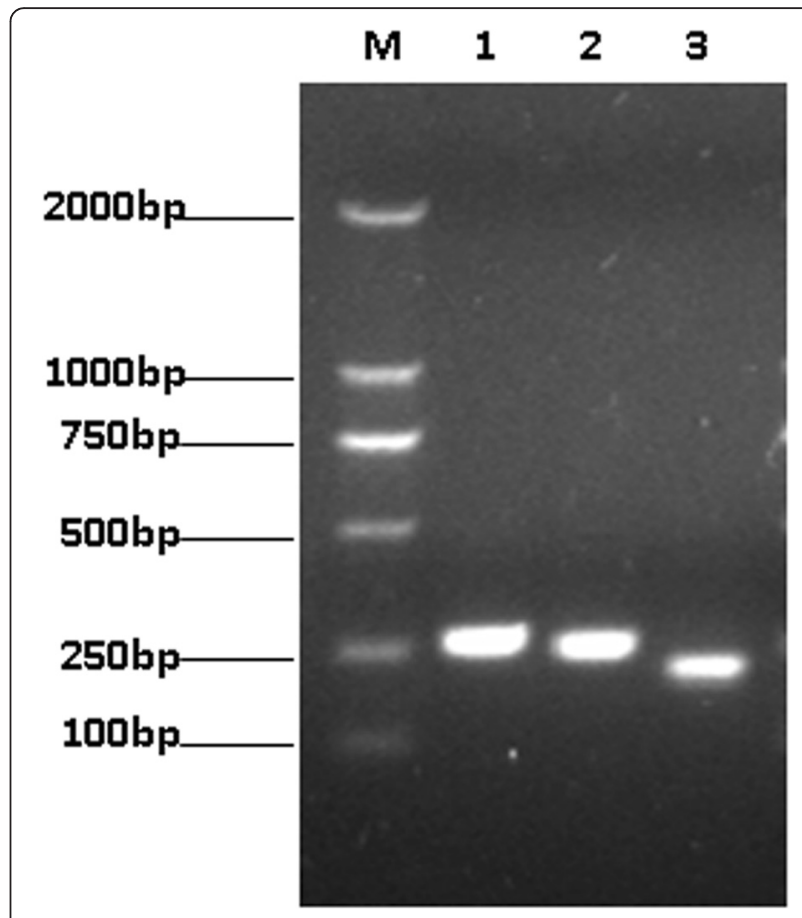

Fig. 1 The nucleic acid fragments of BSjPGM, BSjRAD23-1 and BSjRAD23-2 by PCR. Lane M: Marker DL 2000 DNA Ladder; Lane 1: BSjPGM; Lane 2: BSjRAD23-1; Lane 3: BSjRAD23-2

\section{Expression and purification of the recombinant proteins} All of the recombinant expression plasmids were successfully transformed into $E$. coli BL21 (DE3) and six kinds of crude fusion proteins were successfully expressed except rBSjPGM, rBSjRAD23-2 and rBSjRAD23-2-BSjPGM. The recombinant antigens, rSjPGM, rSjRAD23, rBSjPGMBSjRAD23-1 and rBSjRAD23-1, were expressed in soluble form. The others existed as the form of inclusion body proteins which were dissolved in $8 \mathrm{M}$ urea solution. The expected molecular mass and the sodium dodecyl sulfate-polyacrylamide gel electrophoresis (SDS-

Table 2 Prediction results using online software for SjPGM and SjRAD23

\begin{tabular}{llll}
\hline Online software & & Application & Results (high score peptides) \\
\hline BepiPred 1.0 & SjPGM & B-cell epitopes prediction & $33-39,101-109,119-138$ \\
& SjRAD23 & & $76-123,174-178,194-230$ \\
IEDB Analysis & SjPGM & B-cell epitopes prediction & $29-40,98-110,119-138,197-203$ \\
& SjRAD23 & & $51-59,75-130,173-180,194-230$ \\
IEDB Analysis & SjPGM & Antigenicity scale & 28-38, 93-98, 109-115, 119-129, 139-167, 221-243 \\
& SjRAD23 & & $42-49,58-77,101-128,163-169,192-238,309-330$ \\
T-epitope Designer & SjPGM & T-epitope prediction & $115-123,132-140$ \\
PredictProtein & SjRAD23 & & $59-67,181-189,195-203$ \\
IEDB Analysis & SjPGM & Long loops prediction & $23-32,91-100,116-152$ \\
& SjRAD23 & & $34-43,51-65,72-162,182-230,282-305$ \\
& SjPGM & Accessibility prediction & $82-91,98-104,114-122$ \\
\hline
\end{tabular}




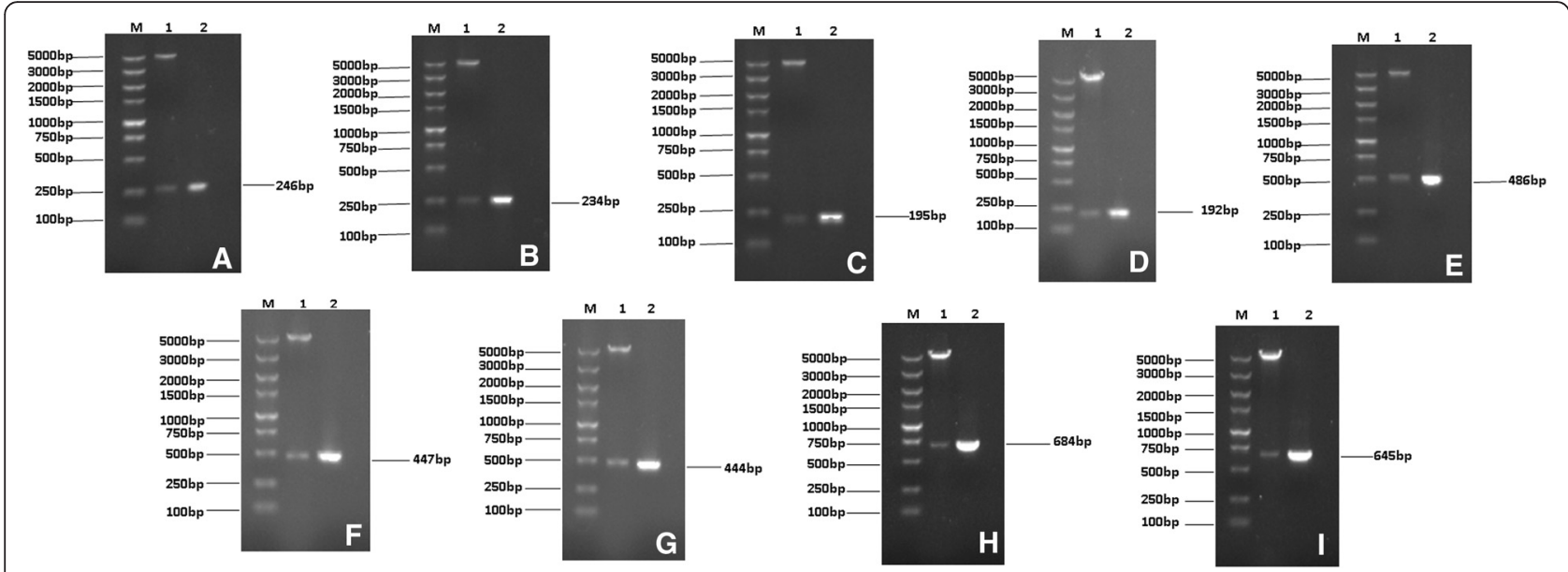

Fig. 2 Analysis of the recombinant plasmids by restriction enzyme digestion and PCR. a, pET-28a (+)-BSjPGM; b, pET-28a (+)-BSjRAD23-1; c, pET28a (+)-BSjRAD23-2; d, pET-32a (+)-BSj23; e, pET-28a (+)-BSjPGM-BSjRAD23-1; f, pET-28a (+)-BSjRAD23-2-BSjPGM; $\mathbf{g}$, pET-28a (+)-BSjPGM-BSj23; $\mathbf{h}$, pET-28a (+)-BSjPGM-BSjRAD23-1-BSj23; i, pET-28a (+)-BSjRAD23-2-BSjPGM-BSj23. Lane 1: The recombinant plasmid digested with restriction enzymes; Lane 2: PCR results using the recombinant plasmid as template; Lane M: Marker DL5000 DNA Ladder

PAGE) of the six recombinant proteins are shown in Table 3 and Fig. 3.

\section{Diagnosis of goat schistosomiasis using recombinant antigens as detecting antigens}

To assess the potential of rLHD-Sj23, rSjPGM, rSjRAD23 and the newly constructed multi-epitope proteins as diagnostic antigens for goat schistosomiasis, sera from 91 schistosome-infected goats, 44 non-infected goats, 12 goats infected with H.contortus and 37 Orientobilharzia-infected goats were tested using these recombinant antigens and SEA (control) as detecting antigen, yielding results shown in Fig. 4 and Table 4. Among the eight recombinant antigens, three showed higher sensitivity with $89.01 \%$ (81/91, $95 \%$ CI: 80.72-94.60\%) for rBSjRAD23-2-BSjPGM-BSj23, 93.41 \% (86/91, 95 \% CI: 87.64-98.19 \%) for rBSjPGM-BSj23 and 97.80\% (89/91, 95 \% CI: 92.29-99.73 \%) for rBSjPGM-BSjRAD23-1BSj23. Most of the multi-epitope antigens showed higher sensitivity than single molecular recombinant antigens rLHD-Sj23, rSjRAD23 and rSjPGM except for rBSjPGM-BSjRAD23-1 (59.34 \%, 95 \% CI: 48.53$69.52 \%)$. The sensitivity of rBSjPGM-BSjRAD23-1-

Table 3 Expected sizes of the recombinant proteins

\begin{tabular}{lll}
\hline The multi-epitope antigens & $\begin{array}{l}\text { Number of } \\
\text { amino acids }\end{array}$ & Molecular mass (kDa) \\
\hline rBSjRAD23-1 & 78 & 16 \\
rBSj23 & 64 & 7 \\
rBSjPGM-BSjRAD23-1 & 162 & 25.8 \\
rBSjPGM-BSj23 & 148 & 22.6 \\
rBSjPGM-BSjRAD23-1-BSj23 & 228 & 34 \\
rBSjRAD23-2-BSjPGM-BSj23 & 215 & 32 \\
\hline
\end{tabular}

BSj23 (97.8 \%) was higher than the other recombinant antigens. The sensitivity of all recombinant antigens was lower than that of SEA (100.0\%, $95 \%$ CI: 96.03-100\%).

The specificity of most of the recombinant antigens was $100 \%(44 / 44,95 \%$ CI: 91.96-100\%) except rBSjPGM-BSjRAD23-1 (97.73 \%, 43/44, 95 \% CI: 87.9899.94 \%) and rBSj23 (93.18 \%, 41/44, 95 \% CI: 81.34$98.57 \%)$ which all were higher than that of SEA (72\%, 33/44, 95 \% CI: 59.66-86.81 \%) (Table 4). These antigens were also used to detect sera from Orientobilharzia-infected goats and $H$. contortus-exposed goats. No crossreactivity with sera from $H$. contortus-exposed goats was observed for most of the recombinant antigens tested except for rSjBPGM-BRAD23-1-BSj23 (8.33 \%, 1/12, $95 \%$ CI: 2.11-38.48 \%) compared with that of SEA (25\%, 3/12, 95 \% CI: 5.49-57.91 \%) (Table 4). Four recombinant antigens (rSjPGM, rSjRAD23, rBSjRAD23-2BSjPGM-BSj23 and rBSjPGM-BSjRAD23-1) have no cross-reactivity with sera from Orientobilharzia-infected goats. The cross-reactivities with orientobilharziasis of other recombinant antigens were $10.82 \%$ (rBSjRAD231, 4/37, 95 \% CI: 3.03-25.42\%), $5.41 \%$ (rBSj23, 2/37, 95 \% CI: 0.66-18.19\%), 2.70 \% (rBSjPGM-BSj23, 1/37, $95 \%$ CI: $0.07-14.16 \%)$ and $13.51 \%$ (rBSjPGMBSjRAD23-1-BSj23, 5/37, 95 \% CI: 4.54-28.77 \%), respectively. As a control, SEA showed a very high crossreactivity (31/37, 83.78 \%, $95 \%$ CI: 67.99-93.81 \%) with sera from Orientobilharzia-infected goats.

\section{Discussion}

It is still a huge challenge to diagnose schistosomiasis in low endemic areas in China after six decades of integrated schistosomiasis control $[21,22]$. Animal 

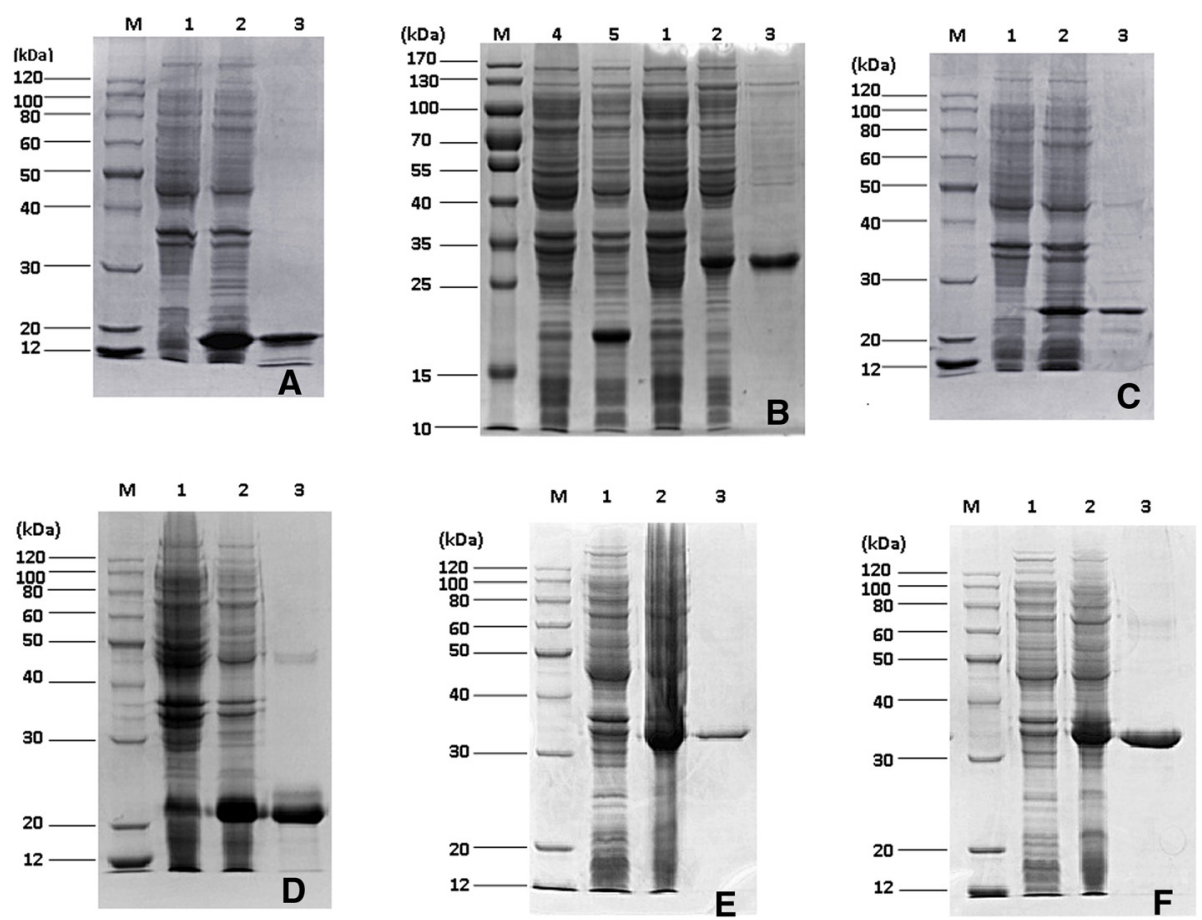

Fig. 3 Expression and purification analysis of recombinant proteins. a, rBSjRAD23-1; b, rBSj23; c, rBSjPGM-BSjRAD23-1; d, rBSjPGM-BSj23; e, rBSjPGM-BSjRAD23-1-BSj23; f, rBSjRAD23-2-BSjPGM-BSj23. Lane M: Molecular markers; Lane 1: Total extract from vectors pET-28a (+) (B: pET-32a (+) vector with no IPTG) after induction with 1 mM IPTG; Lane 2: Total extract from recombination proteins after induction with 1 mM IPTG; Lane 3: The recombinant protein purified through $\mathrm{Ni}^{2+}$-charged column chromatography; Lane 4: Total extract from pET-32a (+) vector; Lane 5: Total extract from from pET-32a (+) vector after induction with $1 \mathrm{mM}$ IPTG

surveillance is a loophole in the transmission of schistosomiasis as there is a lack of importance given to the role of animal hosts [2], especially goats in some endemic regions. As confirmed in previous studies, the sedimentation technique and Kato-Katz or hatching test may not detect light infections in reservoir hosts which have undergone mass drug administration [23-25]. Antibody-detection has been shown to be more sensitive than parasitological techniques and is needed in areas characterised by a low level of transmission, low prevalence and particular low intensity [22, 26]. Although serological diagnosis is not able to discriminate between active and past infections and has higher cross reactions with other parasites [7], many methods based on the detection of antigens or specific antibodies have been developed and applied in the field. Some immunological tests developed in China have acceptable performance characteristics and some serological methods are used largely as a screening tool in China, such as indirect hemagglutination assay (IHA) [27, 28]. Molecular methods, mainly DNA amplification techniques by PCR, based on the genomic DNA of schistosomes are able to detect DNA from all phases of the life-cycle of this parasite $[29,30]$. Although the adequacy and sensitivity of the PCR methods have been proven in schistosome infections in water buffaloes [31] and in other helminth and protozoan infections in humans [32,33], the sensitivity of molecular methods can be affected by the degree of infection and methods have poor agreement [34]. Furthermore, the molecular diagnostic techniques are more expensive than serological diagnostic methods.

Schistosome-infected domestic animals are an important source for the transmission of schistosomiasis [35]. Goats are a source of infection due to the large population sizes, wide range of activities and high infection rates in some schistosomiasis endemic regions. The role of goats in the spread of schistosomiasis may become increasingly prominent and could be a potential hazard, causing a rebound of schistosomiasis in snail habitats if there are no diagnosis measures as in the effective control on infected bovines, patients and their waste [36]. The detection for goat schistosomiasis should warrant further attention and establishing a sensitive and specific diagnostic test is urgently required.

The LHD-Sj23, SjPGM and SjRAD23 were selected to test in this study because they possess higher sensitivity and specificity in detecting water buffalo schistosomiasis $[8,20]$. But when we used these single recombinant molecules as diagnosis antigen to detect goat schistosomiasis, although they all had higher specificity, the sensitivity of 
rSjPGM
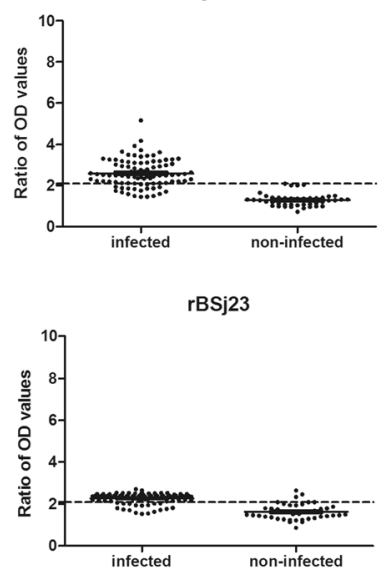

rBSjPGM-BSjRAD23-1-BSj23

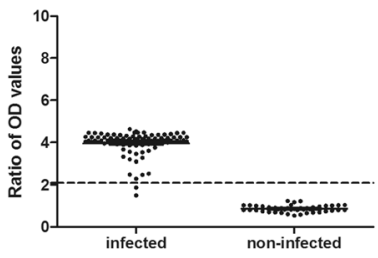

rSjRAD23

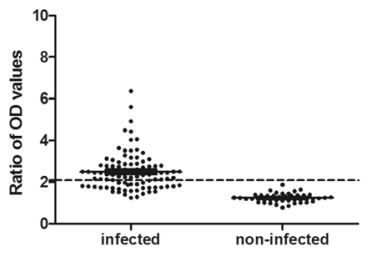

rBSjPGM-BSjRAD23-1

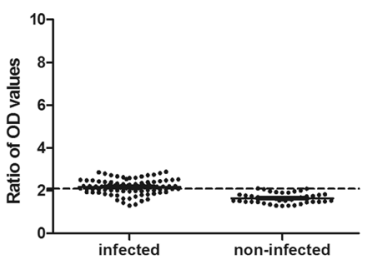

rBSjRAD23-2-BSjPGM-BSj23

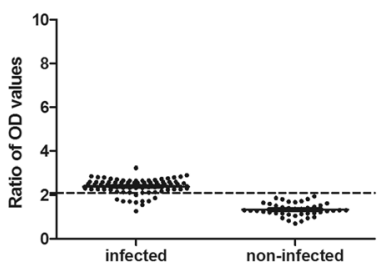

rBSjRAD23-1

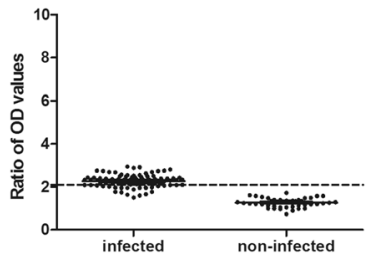

rBSjPGM-BSjRAD23-1

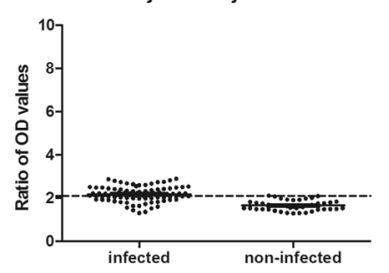

SEA

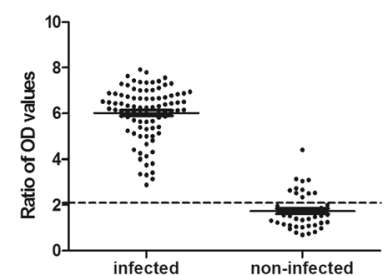

Fig. 4 Analysis of the recombinant proteins and SEA for the diagnosis of goat schistosomiasis by ELISA. Sera from 91 schistosome infected goats and 44 uninfected goats were tested

the ELISA for rSjPGM and rSjRAD23 were only 79.12 and $65.93 \%$, lower than the $91.35 \%(95 / 104)$ and $88.46 \%$ $(92 / 104)$ for an ELISA with the same antigens in water buffalo; this may correlate with species or immune responses difference between goat and water buffalo.

To further increase the sensitivity of the diagnostic technique for goat schistosomiasis, epitopes of SjPGM and SjRAD23 were predicted. Recombinant multiepitope proteins were constructed and their potential as diagnostic antigens for goat schistosomiasis was evaluated in this study. To enhance the accuracy of epitope prediction, several online software analytical methods were applied. The peptides with the highest score in each online method were selected. The peptides that included B-cell epitope-rich regions and possessed higher antigenicity were considered first. According to the conclusion that synthetic peptides containing linear-cell epitopes can be used to raise antibodies against specific proteins and as a diagnostic reagent. B-cell epitopes are the regions by which some proteins or antibodies (produced by B-cells) bind [37]. In the end, two polypeptides (76-123, 194-230) from SjRAD23 and one polypeptide (85-166) from SjPGM were selected. Considering that the LHD-Sj23 is a B-cell epitope-rich region and has been confirmed to be a sensitive diagnostic molecule for goat and cattle schistosomiasis [18-20], three newly predicted polypeptides and the LHD-Sj23 were chosen to construct the recombinant multi-epitope protein expression plasmids. A total of five recombinant plasmids were constructed with four of them being successfully expressed.

Four recombinant proteins, four recombinant multiepitope proteins and SEA were applied as diagnostic antigens to detect goat schistosomiasis. The results show that the sensitivity of three recombinant multi-epitope

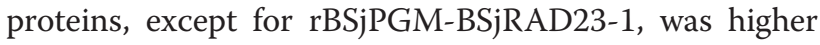
than those of four single-molecule recombinant proteins. Among the three recombinant multi-epitope antigens, rBSjPGM-BSjRAD23-1-BSj23 exhibited the highest sensitivity (97.8\%), slightly lower than SEA (100\%), similarly, the specificity of rBSjPGM-BSjRAD23-1-BSj23 was $100 \%$, significantly higher than that of SEA (75\%). In addition, the cross-reactivity with orientobilharziasis and haemonchosis of rBSjPGM-BSjRAD23-1-BSj23 was 13.51 and $8.33 \%$, also significantly lower than that of SEA (83.78 and $25 \%)$. The other two recombinant multiepitope proteins with higher sensitivity and specificity in diagnosis of goat schistosomiasis were rBSjPGM-BSj23 (93.4 and $100 \%$ ) and rBSjRAD23-2-BSjPGM-BSj23 (89 and $100 \%$ ), both also showing lower cross-reactivity with orientobilharziasis $(2.7 \%$ for rBSjPGM-BSj23 and $0 \%$ for rBSjRAD23-2-BSjPGM-BSj23) and haemonchosis (0 \% for both). The conclusion that constructing multi-epitope antigens can increase the sensitivity of serological detection methods has also been reported in Toxoplasma 
Table 4 Comparison of the sensitivity and specificity of various recombinant antigens in the diagnosis of goat schistosomiasis by

\begin{tabular}{|c|c|c|c|c|c|}
\hline Sera sample & & $\begin{array}{l}\text { S. japonicum-infected } \\
\text { goats }\end{array}$ & Uninfected goats & $\begin{array}{l}\text { H. contortus- } \\
\text { infected goats }\end{array}$ & $\begin{array}{l}\text { Orientobilharzia- } \\
\text { infected goats }\end{array}$ \\
\hline No. of cases & & 91 & 44 & 12 & 37 \\
\hline \multirow[t]{2}{*}{ rSjPGM } & No. of positive & 72 & 0 & 0 & 0 \\
\hline & Positive rate (\%) & 79.12 & 0 & 0 & 0 \\
\hline \multirow[t]{2}{*}{ rSjRAD23 } & No. of positive & 60 & 0 & 0 & 0 \\
\hline & Positive rate (\%) & 65.93 & 0 & 0 & 0 \\
\hline \multirow[t]{2}{*}{ rBSjRAD23-1 } & No. of positive & 67 & 0 & 0 & 4 \\
\hline & Positive rate (\%) & 73.63 & 0 & 0 & 10.82 \\
\hline \multirow[t]{2}{*}{ rBSj23 } & No. of positive & 73 & 3 & 0 & 2 \\
\hline & Positive rate (\%) & 80.22 & 6.82 & 0 & 5.41 \\
\hline \multirow[t]{2}{*}{ rBSjPGM-BSjRAD23-1 } & No. of positive & 54 & 1 & 0 & 0 \\
\hline & Positive rate (\%) & 59.34 & 2.27 & 0 & 0 \\
\hline \multirow[t]{2}{*}{ rBSjPGM-BSj23 } & No. of positive & 86 & 0 & 0 & 1 \\
\hline & Positive rate (\%) & 93.41 & 0 & 0 & 2.70 \\
\hline \multirow[t]{2}{*}{ rBSjPGM-BSjRAD23-1-BSj23 } & No. of positive & 89 & 0 & 1 & 5 \\
\hline & Positive rate (\%) & 97.80 & 0 & 8.33 & 13.51 \\
\hline \multirow[t]{2}{*}{ rBSjRAD23-2-BSjPGM-BSj23 } & No. of positive & 81 & 0 & 0 & 0 \\
\hline & Positive rate (\%) & 89.01 & 0 & 0 & 0 \\
\hline \multirow[t]{2}{*}{ SEA } & No. of positive & 91 & 11 & 3 & 31 \\
\hline & Positive rate (\%) & 100.00 & 25.00 & 25.00 & 83.78 \\
\hline
\end{tabular}

gondii [10, 11]. All above-mentioned show constructing multi-epitope antigens is a worthwhile way.

Compared with the results obtained from SEA, which has been widely employed as the diagnostic antigen in most developed serological detecting methods for schistosomiasis, the application of recombinant antigens as diagnosis antigens significantly increased the specificity of diagnostic techniques in this study. It will help to accurately grasp the epidemic, to assess the effect of drug treatment or prevention, and has great significance in reducing repeated drug use for false-positive animals. The recombinant antigens were convenient to prepare, inexpensive and performed favorably to standardise diagnostic techniques, they therefore have good prospects.

Among the three polypeptides which possessed epitope-rich regions selected from SjPGM and SjRAD23, only BSjRAD23-1 was successfully expressed in E. coli in this study. The ELISA tests showed that the sensitivity of rBSjRAD23-1 (73.63\%) was higher than that of rSjRAD23 (65.93\%), and rBSjPGM-BSjRAD23-1-BSj23 $(97.80 \%)$ was higher than that of rBSjPGM-BSj23 (86\%), suggested that recombinant rBSjRAD23-1 possessed potential value as a goat schistosomiasis diagnosis antigen. The recombinant LHD-Sj23 has proven to be a good diagnostic antigen for schistosomiasis in some previous reports $[20,38]$. In this study, we also noted that the inclusion of the LHD-Sj23 peptide in the recombinant multi-epitope antigens could increase the sensitivity in the diagnosis of goat schistosomiasis. The three top sensitive antigens (rBSjPGM-BSjRAD23-1-BSj23, 97 \%; rBSjPGMBSj23, 93.41 \%; rBSjRAD23-2-BSjPGM-BSj23, 89.01 \%) all possessed the composition of the LHD-Sj23 peptide.

The World Health Organisation has noted that case detection will be a problem when elimination of the disease is at hand [39]. Goats are an important host of S. japonicum and should be involved in animal monitoring system in some endemic areas in China. The study presented here suggests that the application of recombinant multiepitope proteins may increase the sensitivity of diagnostic techniques for schistosomiasis, and that the recombinant antigen rBSjPGM-BSjRAD23-1-BSj23 has the potential to be used as a diagnostic antigen for goat schistosomiasis. More sera samples including schistosome-infected and uninfected goats, as well as other parasite-infected goats sera are needed to perform and confirm this result, especially sera from schistosomiasis in low endemic areas and from animals with low infection intensity.

\section{Conclusion}

In conclusion, three recombinant multi-epitope antigens rBSjPGM-BSj23, rBSjRAD23-2-BSjPGM-BSj23 and rBSj PGM-BSjRAD23-1-BSj23 exhibited higher sensitivity and higher specificity than the four recombinant single molecules, as well as higher specificity and lower cross- 
reactivity than SEA. Among all of the tested recombinant antigens, rBSjPGM-BSjRAD23-1-BSj23 showed the highest sensitivity (97.8 \%) and $100 \%$ specificity, having the potential to be used as a diagnostic antigen for goat schistosomiasis. The study also suggested that the application of recombinant multi-epitope diagnostic antigens may increase the sensitivity of the diagnosis technique.

\section{Competing interests}

The authors declare that they have no competing interests.

\section{Authors' contributions}

$C L, X D, Y S, B J$ performed the experiments. YH, JL designed the study and critically revised the manuscript. $\mathrm{CL}, \mathrm{XC}, \mathrm{TW}, \mathrm{QH}$ performed the statistical analysis. ZF, KL, CZ, HL, RX, ZZ, JZ, JF provided most of materials needed. All authors read and approved the final manuscript.

\section{Acknowledgements}

The authors wish to thank Professor Jianzhi Liu from Tibet Academy of Agriculture and Animal Husbandry Sciences, Professor Xiangrui Li, Professor Xiaokai Song and Yujian Wang from Nanjing Agricultural University for providing goat sera from infections with Orientobilharzia and goat sera from infections with $\mathrm{H}$. contortus.

\section{Author details}

'Key Laboratory of Animal Parasitology, Ministry of Agriculture of China, Shanghai Veterinary Research Institute, Chinese Academy of Agricultural Sciences, Shanghai, China. ${ }^{2}$ College of Life and Environmental Science, Shanghai Normal University, Shanghai, China. ${ }^{3}$ Jiangsu Co-innovation Center for Prevention and Control of Important Animal Infectious Diseases and Zoonoses, Yangzhou, China.

Received: 10 December 2015 Accepted: 1 March 2016

Published online: 09 March 2016

\section{References}

1. Lei ZL, Zheng H, Zhang LJ, Zhu R, Xu ZM, Xu J, et al. Endemic status of schistosomiasis in People's Republic of China in 2013. Chin J Schisto Control. 2014;26(6):591-7 [in chinese].

2. Angeles JM, Goto Y, Kirinoki M, Asada M, Leonardo LR, Rivera PT, et al. Utilization of ELISA using thioredoxin peroxidase-1 and tandem repeat proteins for diagnosis of Schistosoma japonicum infection among water buffaloes. PLoS Negl Trop Dis. 2012;6(8), e1800.

3. He YX, Salafsky B, Ramaswamy K. Host-parasite relationships of Schistosoma japonicum in mammalian hosts. Trends Parasitol. 2001:17:320-4.

4. Li H, Liu JM, Song JX, Ma SH, Wang Q, Heng L, et al. Domestic animal shistosomiasis in 2012 in China. Chin J Anim Infect Dis. 2014;22(5):68-71 [in chinese].

5. Liu J, Zhu C, Shi Y, Li H, Wang L, Qin S, et al. Surveillance of Schistosoma japonicum infection in domestic ruminants in the Dongting Lake region Hunan province, China. PLoS One. 2012;7(2), e31876.

6. Zhou YB, Liang S, Jiang QW. Factors impacting on progress towards elimination of transmission of schistosomiasis japonica in China. Parasit Vectors. 2012;5:275.

7. Xie SY, Yuan M, Ji MJ, Hu F, Li ZJ, Liu YM, et al. Immune responses result in misdiagnosis of Schistosoma japonicum by immunodiagnosis kits in eggpositive patients living in a low schistosomiasis transmission area of China. Parasit Vectors. 2014;7:95

8. Zhang M, Fu Z, Li C, Han Y, Cao X, Han H, et al. Screening diagnostic candidates for schistosomiasis from tegument proteins of adult Schistosoma japonicum using an immunoproteomic approach. PLoS Negl Trop Dis. 2015; 9(2), e0003454.

9. Jin Y, Lu K, Zhou WF, Fu ZQ, Liu JM, Shi YJ, et al. Comparison of recombinant proteins from Schistosoma japonicum for schistosomiasis diagnosis. Clin Vaccine Immunol. 2010;17(3):476-80.

10. Hajissa K, Zakaria R, Suppian R, Mohamed Z. Design and evaluation of a recombinant multi-epitope antigen for serodiagnosis of Toxoplasma gondii infection in humans. Parasit Vectors. 2015;8:315.
11. Dai JF, Jiang $M, Q u$ LL, Sun L, Wang $Y Y$, Gong LL, et al. Toxoplasma gondii: enzyme-linked immunosorbent assay based on a recombinant multiepitope peptide for distinguishing recent from past infection in human sera. Exp Parasitol. 2013;133:95-100.

12. Hong Y, Sun A, Zhang M, Gao F, Han Y, Fu Z, et al. Proteomics analysis of differentially expressed proteins in schistosomula and adult worms of Schistosoma japonicum. Acta Trop. 2013;126(1):1-10.

13. Doenhoff MJ, Butterworth AE, Hayes RJ, Sturrock RF, Ouma JH, Koech D, et al. Seroepidemiology and serodiagnosis of schistosomiasis in Kenya using crude and purified egg antigens of Schistosoma mansoni in ELISA. Trans R Soc Trop Med Hyg. 1993;81(1):42-8.

14. Larsen JE, Lund O, Nielsen M. Improved method for predicting linear B-cell epitopes. Immunome Res. 2006;2:2

15. Emini EA, Hughes JV, Perlow DS, Boger J. Induction of hepatitis A virus-neutralizing antibody by a virus-specific synthetic peptide. J Virol. 1985;55(3):836-9.

16. Kolaskar AS, Tongaonkar PC. A semi-empirical method for prediction of antigenic determinants on protein antigens. FEBS Lett. 1990;276:172-4.

17. Calis JJ, Maybeno M, Greenbaum JA, Weiskopf D, De Silva AD, Sette A, et al. Properties of MHC class I presented peptides that enhance immunogenicity. PLoS Comput Biol. 2013;9(10), e1003266.

18. Reynolds SR, Shoemaker CB, Harn DA. T and B cell epitope mapping of SM23, an integral membrane protein of Schistosoma mansoni. J Immunol. 1992;149(12):3995-4001.

19. Lin JJ. Research of chinese strain Schistosoma japonicum $23 \mathrm{kD}$ recombinant antigen screening encoding 23kD antigen gene clones. Chin J Vet Sci Technol. 1995;25(8):19-20 [in chinese].

20. Lin JJ, Li H, Lu K, Shi YJ, Fu ZQ, Cai YM, et al. Applications sj23 recombinant antigen diagnostic cattle, goat schistosomiasis research. Acta Vet Et Zootechn Sin. 2003;05:506-8 [in chinese].

21. Wang XH, Wu XH, Zhou XN. Bayesian estimation of community prevalences of Schistosoma japonicum infection in China. Int J Parasitol. 2006;36(8):895-902.

22. Xu J, Peeling RW, Chen JX, Wu XH, Wu ZD, Wang SP, et al. Evaluation of immunoassays for the diagnosis of Schistosoma japonicum infection using archived sera. PLoS Negl Trop Dis. 2011;5(1), e949.

23. Kato-Hayashi N, Leonardo LR, Arevalo NL, Tagum MN, Apin J, Agsolid LM, et al. Detection of active schistosome infection by cell-free circulating DNA of Schistosoma japonicum in highly endemic areas in Sorsogon Province, the Philippines. Acta Trop. 2015;141(Pt B):178-83.

24. Lin DD, Liu JX, Liu YM, Hu F, Zhang YY, Xu JM, et al. Routine Kato-Katz technique underestimates the prevalence of Schistosoma japonicum: a case study in an endemic area of the People's Republic of China. Parasitol Int. 2008;57(3):281-6.

25. Han S, Zhang X, Wen J, Li Y, Shu J, Ling H, et al. A combination of the KatoKatz methods and ELISA to improve the diagnosis of clonorchiasis in an endemic area, China. PLoS One. 2012:7(10), e46977.

26. Doenhoff MJ, Chiodini PL, Hamilton JV. Specific and sensitive diagnosis of schistosome infection: can it be done with antibodies? Trends Parasitol. 2004 20(1):35-9.

27. van Dam GJ, Xu J, Bergquist R, de Dood CJ, Utzinger J, Qin Z-Q, et al. An ultra-sensitive assay targeting the circulating anodic antigen for the diagnosis of Schistosoma japonicum in a low-endemic area, People's Republic of China. Acta Trop. 2015;141(Pt B):190-7.

28. Cai YC, Xu JF, Steinmann P, Chen SH, Chu YH, Tian LG, et al. Field comparison of circulating antibody assays versus circulating antigen assays for the detection of schistosomiasis japonica in endemic areas of China. Parasit Vectors. 2014;7:138.

29. Espirito-Santo MC, Alvarado-Mora MV, Dias-Neto E, Botelho-Lima LS, Moreira JP, Amorim M, et al. Evaluation of real-time PCR assay to detect Schistosoma mansoni infections in a low endemic setting. BMC Infect Dis. 2014:14:558.

30. Cnops L, Tannich E, Polman K, Clerinx J, Van Esbroeck M. Schistosoma realtime PCR as diagnostic tool for international travellers and migrants. Trop Med Int Health. 2012;17(10):1208-16.

31. Wu HW, Qin YF, Chu K, Meng R, Liu Y, McGarvey ST, et al. High prevalence of Schistosoma japonicum infection in water buffaloes in the Philippines assessed by real-time polymerase chain reaction. Am J Trop Med Hyg. 2010; 82(4):646-52.

32. Wongratanacheewin S, Pumidonming W, Sermswan RW, Pipitgool V, Maleewong W. Detection of Opisthorchis viverrini in human stool specimens by PCR. J Clin Microbiol. 2002;40(10):3879-80.

33. Moon JH, Cho SH, Yu JR, Lee WJ, Cheun HI. PCR diagnosis of Entamoeba histolytica cysts in stool samples. Korean J Parasitol. 2011;49(3):281-4. 
34. Lier T, Simonsen GS, Haaheim H, Hjelmevoll SO, Vennervald BJ, Johansen MV. Novel real-time PCR for detection of Schistosoma japonicum in stool. Southeast Asian J Trop Med Public Health. 2006;37(2):257-64.

35. Shi YJ, Li H, Lu K. Schistosomiasis domestic animals were the source of the first transmission. Chin J Vet Parasitol. 2004;7(3):41-2 [in chinese].

36. Wang QZ, Wang TP, Zhang SQ. Research progress on transmission capacity of reservoir host of Schistosoma japonicum. Chin J Schisto Control. 2013;32: 86-9 [in chinese].

37. Schellekens GA, Visser $H$, de Jong BA, van den Hoogen FH, Hazes JM, Breedveld FC, et al. The diagnostic properties of rheumatoid arthritis antibodies recognizing a cyclic citrullinated peptide. Arthritis Rheum. 2000; 43(1):155-63.

38. Li Y, Wang L, Fang R, Nie H, Zhou Y, Zhao J, et al. Establishment and evaluation of an iELISA using the recombinant membrane protein LHD-Sj23 for the serodiagnosis of Schistosoma japonicum infection in cattle in China. Vet Parasitol. 2012;188(3-4):247-54.

39. World Health Organization. Elimination of schistosomiasis from low transmission areas: Report of a WHO informal consultation. Salvador, Bahia, Brazil; 2008. Geneva,2009;2.(WHO/HTM/NTD/PCT/2009;2. Available at: http:// apps.who.int/iris/bitstream/10665/70127/1/WHO_HTM_NTD_PCT_2009.2_ eng.pdf).

\section{Submit your next manuscript to BioMed Central} and we will help you at every step:

- We accept pre-submission inquiries

- Our selector tool helps you to find the most relevant journal

- We provide round the clock customer support

- Convenient online submission

- Thorough peer review

- Inclusion in PubMed and all major indexing services

- Maximum visibility for your research

Submit your manuscript at www.biomedcentral.com/submit 